\title{
Olfactory ensheathing cells, olfactory nerve fibroblasts and biomatrices to promote long-distance axon regrowth and functional recovery in the dorsally hemisected adult rat spinal cord
}

\author{
R. Deumens ${ }^{\text {a }}$, G.C. Koopmans ${ }^{\text {a,b }}$, W.M.M. Honig ${ }^{\text {b }}$, F.P.T. Hamers ${ }^{\text {c }}$, V. Maquet ${ }^{\text {d }}$, R. Jérôme ${ }^{\text {d }}$, H.W.M. \\ Steinbusch $^{\text {a }}$, E.A.J. Joosten ${ }^{b}$ \\ ${ }^{a}$ Department of Psychiatry and Neuropsychology, Division Neuroscience, European Graduate School of \\ Neuroscience (EURON), University of Maastricht, P.O. Box 616, 6200 MD, Maastricht, The Netherlands \\ ${ }^{b}$ Department of Anesthesiology, Academic Hospital Maastricht, P.O. Box 5800, 6202 AZ, Maastricht, The \\ Netherlands \\ ${ }^{c}$ Department of Physical Medicine and Rehabilitation, Rudolf Magnus Institute for Neuroscience, University \\ Medical Center Utrecht, Utrecht, The Netherlands Center for Education and Research on Macromolecules, \\ University of Liège, Sart-Tilman B6, 4000 Liège, Belgium
}

\begin{abstract}
Cellular transplantation, including olfactory ensheathing cells (OEC) and olfactory nerve fibroblasts (ONF), after experimental spinal cord injury in the rat has previously resulted in regrowth of severed corticospinal (CS) axons across small lesion gaps and partial functional recovery. In order to stimulate CS axon regrowth across large lesion gaps, we used a multifactorial transplantation strategy to create an OEC/ONF continuum in spinal cords with a 2-mm-long dorsal hemisection lesion gap. This strategy involved the use of aligned OEC/ONF-poly(D,L)lactide biomatrix bridges within the lesion gap and OEC/ONF injections at $1 \mathrm{~mm}$ rostral and caudal to the lesion gap. In order to test the effects of this complete strategy, control animals only received injections with culture medium rostral and caudal to the lesion gap. Anatomically, our multifactorial intervention resulted in an enhanced presence of injured CS axons directly rostral to the lesion gap $(65.0 \pm 12.8 \%$ in transplanted animals versus $13.1 \pm 3.9 \%$ in control animals). No regrowth of these axons was observed through the lesion site, which may be related to a lack of OEC/ONF survival on the biomatrices. Furthermore, a 10-fold increase of neurofilament-positive axon ingrowth into the lesion site as compared to untreated control animals was observed. With the use of quantitative gait analysis, a modest recovery in stride length and swing speed of the hind limbs was observed. Although multifactorial strategies may be needed to stimulate repair of large spinal lesion gaps, we conclude that the combined use of $\mathrm{OEC} / \mathrm{ONF}$ and poly $(\mathrm{D}, \mathrm{L})$-lactide biomatrices is rather limited.
\end{abstract}

Keywords: Regeneration; Rat; Locomotion; Olfactory glia; Polylactides; Tracing; Cell survival; Migration; Plasticity; Combinatory

\section{Introduction}

Axonal disruption is the key factor with respect to functional loss after spinal cord injury (SCI). Spontaneous regeneration of severed axons does not occur, mainly because of the injury-induced disbalance between growthpromoting and growth-inhibiting factors in the spinal cord environment (Fawcett, 1997). Transplantation of growth-promoting grafts has been performed extensively in order to modulate this balance under experimental conditions and thereby stimulate axon regrowth and functional recovery (Deumens et al., 2005). In the last decade, mixed cultures of olfactory ensheathing cells (OEC) and olfactory nerve fibroblasts (ONF) and purified OEC cultures have been extensively tested as a possible therapeutic tool in experimental SCI research (Raisman, 2001). Physiologically, OEC reside in the mammalian olfactory system, where they stimulate olfactory axon regeneration throughout the lifespan of the organism (Ramon-Cueto and Avila, 1998), a process which is thought to be positively influenced by the presence of ONF (Raisman, 2001). When transplanted acutely into incomplete/complete rat spinal lesion sites, mixed OEC/ONF or purified OEC have stimulated [1] regrowth of axons from various injured tracts, such as the corticospinal (CS) (Li et al., 1997, 1998), raphespinal (Lu et al., 2001), reticulospinal (Plant et al., 2003), and coerulospinal tract (Ramon-Cueto et al., 2000), [2] restoration in the signal conduction across the lesion/ graft site (Imaizumi et al., 2000), and [3] recovery of body functions, such as locomotor functions ( $\mathrm{Li}$ et al., 1998), respiratory functions (Li et al., 2003), and sensory functions (Ramon-Cueto et al., 2000). 
The CST is known to have a low regenerative response after injury (Joosten, 1997). Although regrowth of injured CS axons has been stimulated after transplantation of mixed OEC/ONF and purified OEC cultures into the injured spinal cord tissue, the spinal cord injury models usually included small spinal lesion gaps. In the injured human spinal cord, large cystic cavities may develop, requiring long-distance axon regrowth. Longdistance axon regeneration of olfactory axons is suggested to be associated by both OEC and ONF (Li et al., 2005). Therefore, we hypothesized that transplanted OEC/ONF cultures promote repair of injured CS axons across large spinal lesion gaps. However, regrowth of injured CS axons across large lesion gaps may not only require a growth-stimulating source, such as $\mathrm{OEC} / \mathrm{ONF}$, but also a physical substrate to bridge the lesion gap. In order to avoid the use of millions of autologous growth-promoting cells, other bridges have been developed, such as growth-promoting tissues (Coumans et al., 2001), growth-promoting cells secreting extracellular matrix molecules (Li et al., 2003), and organic bridges (Schnell and Schwab, 1993).

In the present study, a multifactorial transplantation strategy including a physical bridge was used to create an OEC/ONF continuum in rat spinal cords with large lesion gaps. Since dorsal hemisection is often used to study CS axon regrowth (Schnell and Schwab, 1990; Schnell et al., 1994; Grill et al., 1997; GrandPre et al., 2002), we used a 2-mm-long dorsal hemisection lesion. Aligned OEC/ONF-biomatrix complexes, which have been shown to stimulate directional neurite growth in vitro (Deumens et al., 2004), were acutely transplanted into the 2-mmlong dorsal hemisection lesion gaps. In addition, OEC/ONF suspensions were injected rostral and caudal to the thoracic lesion site. Migration of OEC/ONF after transplantation into the injured spinal cord, as has been previously reported (Li et al., 1997, 1998), may then create a continuum of OEC/ONF in the injured spinal cord. In order to study the effect of this multifactorial treatment including OEC/ONF-biomatrix complexes and OEC/ONF cell injections, control animals did not receive a graft within the lesion site and received culture medium injections rostral and caudal to the injury site. We hypothesize that our multifactorial transplantation strategy stimulates regrowth of injured CS axons across the large spinal lesion site and subsequently recovery of impaired body functions.

\section{Materials and methods}

\section{General}

All experimental procedures were performed according to the recommendations of the European Commission (European Communities Council Directive of 24 November 1986; 86/609/ EEC), and protocols were approved by the Committee on Animal Research of the Maastricht University (DEC 2004-030). In this study, every attempt was made to minimize the number of animals and their suffering. Male Lewis rats (inbred, animal facilities of Maastricht University), 7 weeks old, 200-250 g in body weight, were pretrained for CatWalk behavioral testing (Hamers et al., 2001; Koopmans et al., 2005) until they were capable of making three consecutive runs without hesitation. At the age of 9 weeks, the animals were behaviorally tested on the CatWalk to obtain preoperative CatWalk data. Next, a dorsal hemisection of the spinal cord was performed at T11/T12, and animals were randomly divided into two groups; one group $(n=10)$ received OEC/ONF-biomatrix transplants into the lesion site and OEC/ONF suspensions into the rostral and caudal cord stumps, and the other group $(n=8)$ did not receive transplants into the lesion site, but received injections of culture medium into the rostral and caudal host stumps. After this, the animals were tested behaviorally using the Basso-BeattieBresnahan locomotor rating scale (Basso et al., 1995) and the CatWalk gait analysis for 9 weeks. In order to label the CS tract, an additional surgery was performed at 6 weeks after injury. Nine weeks after SCI, the animals were sacrificed and processed for histological analysis. Throughout the postoperative period and during histological analysis, the researchers were blind to the treatment.

\section{OEC/ONF cultures}

The isolation of mixed OEC/ONF cultures containing syngeneic cells from the outer two glomerular layers of adult rat olfactory bulbs was identical to that described previously ( $\mathrm{Li}$ et al., 1998) and is based upon a method developed by Ramon-Cueto and co-workers (Ramon-Cueto and Nieto-Sampedro, 1992). Briefly, 9-week-old Lewis rats (inbred; animal facilities of Maastricht University) were decapitated, and the olfactory bulbs were dissected and cleared of meninges. Then, the outer olfactory nerve fiber and glomerular layers were carefully dissected. The tissue was diced in small fragments and incubated with trypsin $(0.1 \%$ in phosphate-buffered saline; Gibco/Invitrogen, Breda, The Netherlands) at $37^{\circ} \mathrm{C}$ for $15 \mathrm{~min}$. Trypsinization was stopped by addition of culture medium. Dulbecco's Modified Eagle's medium (DMEM/NUT mix F12; Gibco/Invitrogen, Breda, The Netherlands) with glutamax-I supplemented with $10 \%$ inactivated fetal calf serum (Bodinco, Alkmaar, The Netherlands) and antibiotics (100 U/ml penicillin and $100 \mu \mathrm{g} / \mathrm{ml}$ streptomycin) was the only culture medium used in this study. The tissue was washed twice with culture medium and collected in $1 \mathrm{ml}$ culture medium. 
Then, single cell dissociation was achieved by mechanical trituration. The cells were plated in PLL-coated Petri dishes at a density of 200,000 cells in $2 \mathrm{ml}$ of culture medium per Petri dish. The cells were grown for 4-5 days before half of the culture medium was refreshed. Thereafter, the culture medium was refreshed every 2 days. It is known that this protocol renders mixed cultures containing both OEC and ONF (Li et al., 1997; Deumens et al., 2004). Therefore, these cultures will be named OEC/ONF cultures.

\section{Poly(D,L)-lactide matrices}

Poly(D,L)-lactide with inherent viscosity of $1.62 \mathrm{dl} / \mathrm{g}$ was purchased from Purac Biochem (Gorinchem, The Netherlands). A diblock poly(D,L-lactide- $b$-polyethylene oxide) (PLA- $b$-PEO) copolymer with a PLA block of approximately 4000 of molecular weight was synthesized by ring-opening polymerization of D,L-lactide from PEO-monomethyl-ether (with molecular weight ca. approximately 5000, supplied by Sigma, Uithoorn, The Netherlands). Polymer foams were prepared from a mixture of PLA containing $10 \mathrm{wt}$ \% of PLA- $b$-PEO amphiphilic copolymer (PLA/PLA- $b$-PEO). Polymer foams with an aligned pore structure were prepared by freeze-drying of polymer solutions, as previously described (Maquet et al., 2001). The polymer was dissolved in dimethylcarbonate to yield a $5 \mathrm{wt}$.\% polymer solution. The solution was rapidly frozen by immersion into liquid nitrogen in order to induce solvent crystallization. Then, the frozen system was connected to a vacuum pump, and the solvent was removed by sublimation at $-10^{\circ} \mathrm{C}$ and $10^{-2}$ Torr. After $48 \mathrm{~h}$, the drying temperature was increased to $0^{\circ} \mathrm{C}$ and the drying process was continued. The foams were finally dried at room temperature. Small polymer rods ( $1 \times 1 \times 10 \mathrm{~mm}$ ) were cut from the freeze-dried foams using a razor blade and sterilized by UV exposure for $15 \mathrm{~min}$.

\section{OEC/ONF on poly $(D, L)$-lactide matrices}

OEC/ONF cultures that were grown in vitro for 10 days (Days in vitro 10; DIV 10) were collected from the Petri dishes by a 5 -min incubation in $0.1 \%$ trypsin/0.05 mM EDTA (Fluka BioChemika, Buchs, Switzerland) solution at $37^{\circ} \mathrm{C}$. Trypsiniza-tion was stopped using culture medium. After a 10 -min centrifugation at $1200 \mathrm{rpm}$, the pellet was resuspended in culture medium. OEC/ONF cultures were replated onto the surface area of the PLA/PLA- $b$-PEO matrices at a density of about 40,000 cells per $\mathrm{cm}$ and grown on these substrates for 4 days. This protocol renders aligned OEC/ONF-biomatrix complexes as described previously (Deumens et al., 2004).

\section{Prelabeling of OEC/ONF before transplantation}

One day before transplantation, both the OEC/ONF cultures and the OEC/ONF-biomatrix complexes were prelabeled with Hoechst. This was done by a 40- to 60 -min incubation at $37^{\circ} \mathrm{C}$ in $2 \mu \mathrm{g} / \mathrm{ml}$ Hoechst 33342 (Sigma, Uithoorn, The Netherlands) in culture medium. Thereafter, the OEC/ONF cultures and OEC/ONFbiomatrices were extensively washed with culture medium.

\section{Surgeries}

\section{General}

For every operation (i.e. SCI, transplantation, and CS tract labeling), animals received intraperitoneal (i.p.) injections with Buprenorfine (Temgesic $0.1 \mathrm{ml} /$ animal; Schering-Plough, Utrecht, The Netherlands). One hour after the injection, the animals were anesthetized with a mixture of 1-2\% halothane (Abbott, Hoofddorp, The Netherlands) and $\mathrm{O}_{2} / \mathrm{N}_{2} \mathrm{O}(1: 2)$ and an ophthalmic ointment, Visagel (Eurovet, Bladel, The Netherlands), was applied to the eyes to prevent drying during the operation. At the beginning of every operation, the hair overlying the operation area was shaved and the skin was scrubbed with Bethadine. At the end of every operation, the muscles and skin were sutured and animals were observed until awake and then returned to their home cages. At least $1 \mathrm{~h}$ after their awakening, the animals received another i.p. injection with Buprenorfine.

\section{SCI, transplantation}

Laminectomy was performed, and the spinal cord was exposed at T11/T12. The dura mater was cut and with the use of microscissors the dorsal half of the spinal cord was incised at two spinal levels, $2 \mathrm{~mm}$ apart. Using an aspiration device, the dorsal part of the spinal cord in between the two cuts was removed. The depth of the lesion was $1.2 \mathrm{~mm}$, and care was taken to completely remove the dorsal part of the CS tract located in the ventral-most part of the dorsal funiculus. This lesion interrupts descending rubrospinal axons, the dorsal component of the CS tract, all ascending dorsal column axons, and local spinal sensory neurites. The experimental group consisted of 10 animals receiving an OEC/ONF-biomatrix transplant into the thoracic lesion site which was then covered 
with DuraFilm. In addition, OEC/ONF suspensions (100,000 cells/ $\mu 1 ; 2 \mu l$ per injection site; 1 injection site/stump) were injected into the rostral and caudal cord stumps at a distance of $1 \mathrm{~mm}$ from the lesion site and at a depth of $1.2 \mathrm{~mm}$. The control group consisted of 8 animals receiving injections of culture medium into the rostral and caudal cord stumps ( $2 \mu \mathrm{l}$ per injection site) at a distance of $1 \mathrm{~mm}$ from the lesion site and at a depth of $1.2 \mathrm{~mm}$. These culture injections were done to control for possible damage induced by the injection procedure. The control animals did not receive a transplant into the lesion site.

\section{CS tract labeling}

A dental drill was used to make burr holes on both sides of the cranium overlying the sensorimotor cortices. The anterograde neuronal tracer biotin dextran amine (BDA; MW 10,000; Molecular Probes/Invitrogen, Breda, The Netherlands) was slowly injected into the sensorimotor cortex at a depth of $2 \mathrm{~mm}$ from the cortical surface on both sides (10\% in $0.1 \mathrm{M}$ phosphate-buffered saline (PBS), $\mathrm{pH} 7.2 ; 1.5 \mu 1 / 3$ injections/ hemisphere). The needle was left in place for $1 \mathrm{~min}$ and was then gradually withdrawn.

\section{Histological analysis}

At 9 weeks after SCI/3 weeks after CS tract tracing, the rats received an overdose of Nembutal ( $150 \mathrm{mg} / \mathrm{kg}$ body weight; i.p. injection). They were transcardially perfused with ice-cold $4 \%$ paraformaldehyde in $0.1 \mathrm{M}$ phosphate buffer ( $\mathrm{pH}$ 7.4). After perfusion, the spinal cords and the brains were removed and post-fixed in cold $4 \%$ buffered paraformaldehyde overnight. The following day, the tissue was transferred to $10 \%$ sucrose in PBS $(0.1$ $\mathrm{M}$; pH 7.6) and kept overnight at $4^{\circ} \mathrm{C}$. Then, the tissue were transferred to $25 \%$ sucrose in PBS and kept for 3 days at $4{ }^{\circ} \mathrm{C}$. After this cryo-protection procedure, the brain and $2.5-\mathrm{cm}$-long spinal cord pieces including the lesion site were frozen and stored at $-80^{\circ} \mathrm{C}$. Using a cryostat, the spinal cord pieces were serially cut (sagittal sections of $25 \mu \mathrm{m}$ ). In this way, a total of about 120 sections were obtained per spinal cord. The sections were immediately mounted on gelatin chrome-alumn (Sigma, Uithoorn, The Netherlands)-coated glass slides and stored at $-20^{\circ} \mathrm{C}$

Alternate sections were processed for immunohistochemis-try using the following antibodies: mouse anti-p ${ }^{75}$ NGF receptor (1:10,000; Chemicon, Hampshire, UK), mouse anti-neurofilament RT97 (anti-RT97; 1:100; Hybridoma Bank, Iowa City, IA, USA), rabbit anti-GFAP (1:1000; DAKO), rabbit anti-GAP43/B50 (generous gift from Leo van Halewijn, Utrecht University, The Netherlands), and rabbit-serotonin (Prof. Dr. H. Steinbusch, Maastricht University, The Netherlands). Secondary antibodies used were streptavidin-Cy3 (1:2000; for the BDA-labeled CS axons; Jackson ImmunoR-esearch Europe Ltd., Cambridgeshire, UK), Cy3-conjugated donkey anti-mouse (1:800; Jackson ImmunoResearch Europe Ltd., Cambridgeshire, UK), Alexa 488-conjugated goat anti-mouse (1:100; Molecular Probes/Invitrogen, Breda, The Netherlands), and Alexa 488-conjugated goat antirabbit (1:100; Molecular Probes/Invitrogen, Breda, The Netherlands). Antibodies were diluted in 0.3\% Triton X100 in Tris-buffered saline (TBS-T). All primary antibody incubations were overnight at room temperature. All secondary antibody incubations were $1.5 \mathrm{~h}$ at room temperature. In double staining protocols, the primary antibodies were used in a mixture and the incubation with the Cy3-labeled secondary antibody always preceded the incubation with the Alexa-labeled secondary antibody. Before all antibody incubations, the washing steps consisted of 10 min TBS-T, 10 min TBS (TBS-T without Triton X -100), and 10 min TBS-T. All other washing steps involved three times 10 min TBS. For Hoechst stainings, a 30-min incubation in Hoechst 33342 (Sigma, Uithoorn, The Netherlands) was used. Every sixth section was stained for $\mathrm{p}^{75}$, or double stained for $\mathrm{p}^{75}$ and Hoechst, GFAP/ BDA-labeled CS axons, GAP43/BDA-labeled CS axons, GFAP/ neurofilament, or GFAP/serotonin. Stained sections were analyzed using an Olympus AX-70 microscope using epifluo-rescent illumination. The microscope was equipped with a x20 objective and a x10 projection lens. The signal for Alexa 488 (green) was detected using a narrow band MNIBA-type FITC filter (Chroma Technology Corp, Rockingham, VT, USA); the signal for Cy3 (red) was detected using a MNG filter (Chroma Technology Corp, Rockingham, VT, USA); and the signal for Hoechst (blue) was detected using a U-MNIBA filter (Chroma Technology Corp, Rockingham, VT, USA). Pictures of the stained sections were made at a magnification of $\mathrm{x}$ using a Sony Power HAD 3CCD Color Video Camera or an Olympus F-view cooled CCD camera (Paes, Zoeterwoude, The Netherlands). All pictures were analyzed with the image analyzing system analySIS Vers. 3.0. (Soft Imaging System, Munster, Germany). 


\section{Quantitative analysis}

\section{CS axon growth}

All sections with BDA-labeled CS axons were analyzed. For quantitative analysis, $250-\mu \mathrm{m}$-wide boxes were placed at four different locations in the spinal cord sections (see also indications in Fig. 4). The first box was placed in the dorsal gray/white matter at 2.5-3 mm rostral to the injury site and will be termed "the rostral reference". The second box was placed in the dorsal gray/white matter directly rostral to the injury site. The third box was placed in the gray matter underneath the lesion site. Since only few axons were present underneath the lesion site, the box was placed in the area where most BDA immunoreactivity was found. The fourth box was placed in the gray matter caudal to the injury site, again at the position where most BDA immunoreactivity could be found. The few BDA-immuno-reactive CS axons were exclusively present within the caudal gray matter within $10 \mathrm{~mm}$ from the caudal edge of the lesion site. All four boxes were analyzed in all the sections containing BDA-labeled CS axons. The percentage of each box occupied by BDA-immunoreactive axons ("BDA immunoreactivity occupation") was measured, and the box values in all the sections were summed. Since the efficiency of BDA CS tract tracing was highly variable among animals, the BDA immunoreactivity at the rostral reference was taken as the $100 \%$ reference value of BDA-labeled axons of each animal. The BDA immunoreactivity values of boxes 2,3 , and 4 were expressed relative to that of the rostral reference. A similar quantification method has been described previously by others (Goldshmit et al., 2004).

\section{Double staining CS axons and GAP43/B50}

All sections double-stained for CS axons and GAP43/B50 were used to assess whether BDA-labeled CS axons were sprouting/regrowing in the transplanted and/or control animals.

\section{Axon growth into the lesion/graft site}

Every sixth section of five transplanted and five control animals was double-stained for GFAP and neurofilament and used to analyze the degree of neurofilament-positive axon growth into the lesion/graft site. Similar to the quantitative analysis of the BDA-labeled CS tract, the neurofilament immunoreactivity within the total lesion/graft site was measured. GFAP staining was used to correctly delineate the lesion/ graft site. Hence, the percentage of the total lesion/graft site occupied by neurofilament immunoreactivity ("NF immunoreactivity occupation") was measured, summed for all sections, and compared between transplanted and control animals. In order to investigate whether raphespinal axons were among the axons penetrating the graft/lesion site, a double staining of GFAP/serotonin was performed.

\section{Functional testing}

\section{Basso-Beattie-Bresnahan (BBB) locomotor rating scale}

This locomotor test assesses locomotor performance of the hind limbs via a score that ranges from 0 to 21 (Basso et al., 1995). The BBB test was performed according to the recommendations with an observation time of approximately $4 \mathrm{~min}$ and by two investigators which were blind to the treatment. The scores of the left and right hind limbs were averaged and taken as the BBB score of the animal. The BBB test was performed preoperatively at the day of the operation and at the following days postoperatively (DPO): 1, 2, 3, 7, 14, 21, 28, 35, 42, 49, 56, and 63 .

\section{CatWalk gait analysis}

This locomotor test allows an easy and objective analysis of both static and dynamic locomotor parameters, such as stride length, base of support, interlimb coordination, and swing/ stance phases. Details on the CatWalk set-up can be found elsewhere (Hamers et al., 2001 ; Koopmans et al., 2005). Briefly, the animals are trained for about 2 weeks to cross a glass runway without hesitation. During this training period, the animals were motivated for the CatWalk test via a $12 \mathrm{~g}$ /day food restriction protocol. At $24 \mathrm{~h}$ before testing, the animals were deprived of food. Small food pellets (Noyes Precision pellets PJPPP-0045; Sandown Chemical Ltd., Hampton, UK) located at the end of the glass runway were used as a reward. For analysis, three uninterrupted runs were used. 


\section{CatWalk-based BBB scale}

As previously described (Koopmans et al., 2005), the coordination parameter obtained from the CatWalk, the regularity index (RI), can be implemented into the BBB scoring. In brief, the RI grades the degree of coordination as the number of normal step sequence patterns multiplied by four, divided by the total number of paw placements and multiplied by $100 \%$ (Koopmans et al., 2005). Crossings of the glass runway with an RI of $100 \%$ are considered coordinated. If three, two, one, or none of the CatWalk runs is coordinated, consistent, frequent, occasional, or no coordination will be implemented into the BBB score of the animal at that testing day, respectively (Koopmans et al., 2005).

\section{Results}

\section{OEC/ONF cultures}

The mixed OEC/ONF cultures were identical to those described previously (Li et al., 1998). The cultures contained two main cell populations: the OEC and the ONF The OEC population contained elongated, spindleshaped cells co-expressing $\mathrm{p}^{75}$-NGF receptor and S1OO $\beta$. Furthermore, OEC expressed the GFAP protein, either diffusely or intensely. The ONF population consisted of multiple cell types with a flattened fibroblast-like morphology. Immunoreactivity for fibronectin and GFAP in the ONF population suggested the presence of fibroblasts and astrocytes, respectively. Depending on the time

in culture, the relative contribution of OEC/ONF decreased (Deumens et al., 2004), which may reflect either a higher ONF proliferation as compared to OEC or a down-regulation of the $\mathrm{p}^{75}$-NGF receptor by OEC with advancing time in the presence of serum in the culture medium as previously suggested (Fran-ceschini and Barnett, 1996). At the time of transplantation (i.e. after 14 days in vitro), the cultures contained about $10 \%$ OEC, based on selective immunostaining for either S100 $\beta$ or $\mathrm{p}^{75}$-NGF receptor. Prelabeling of the OEC/ONF cultures with Hoechst 1 day prior to transplantation was successful as all the cells were recognizable by the presence of a stained (blue) nucleus (Fig. $1 \mathrm{~A}$ ).

\section{SCI, transplantation}

Eighteen animals received a 2-mm-long dorsal hemisection injury at T11/T12 (Fig. 2). Ten animals received acutely an OEC/ONF-biomatrix that completely filled the lesion gap (Fig. 2) and additionally received OEC/ONF injections into the host tissue rostral and caudal to this lesion gap. Eight control animals only received culture medium injections at the same locations in the host tissue. Although all eighteen animals showed a significant drop in body weight (about 5\%) in the first week postoperatively, they all gained body weight in the weeks thereafter. Furthermore, the two groups of animals did not display a significant difference in body weight at any time point in the study (data not shown).

\section{Transplants within the spinal cord}

At 9 weeks after the SCI and acute transplantation or control intervention, the animals were sacrificed. At the time of dissecting the paraformaldehyde-fixed spinal cords from the transplanted animals, the OEC/ONFbiomatrix complexes were clearly visible at the lesion sites. Furthermore, a stiff connective tissue bridge covered the graft/lesion site. This connective tissue complicated the procedure of taking the fixated spinal cord out of the animals. Although this procedure was carried out with much care, histological analysis of sagittal spinal cord sections showed that the OEC/ONF-biomatrix complex was to a large extent disconnected from the host spinal tissue in many animals. Although a disconnection was often observed between the complex and the underlying host spinal tissue, a disconnection between the complex and the rostral and caudal host tissue was noted in nearly all transplanted animals. Furthermore, many complex remnants were observed within the inner of the complexes, suggesting partly complex degradation. In control animals, the lesion site was devoid of any tissue. Transplanted Hoechst-prelabeled OEC/ONF could easily be found at the levels of the rostral and caudal injection sites, but none could be detected on the biomatrix complexes. The Hoechst-prelabeled OEC/ONF rostral and caudal to the lesion gap were condensed within a relatively small area of the spinal cord, presumably around the injection sites, with little or no migration into the surrounding host tissue (Figs. 1B-D). Although many yellowish crystalloid autofluorescent macrophages (Bunn et al., 2002) were observed in the host spinal cord tissue, viable transplanted Hoechst-prelabeled OEC/ONF could be distinguished from those macrophages because of their blue fluorescence under UV illumination. In addition, a subpopulation of the transplanted Hoechst-prelabeled OEC/ONF was immunoreactive for the $\mathrm{p}^{75}$-NGF receptor (Fig. 1D). In both transplanted and control animals, small, but clearly visible cavities (with a diameter of about $100 \mu \mathrm{m}$ ) were often observed rostral and caudal to 
the injury site to a similar extent in all animals, which may be the result of the injection procedure as they were located at approximately $1 \mathrm{~mm}$ rostral and caudal to the lesion gap. Although no transplanted Hoechst-prelabeled OEC/ONF were detected at or directly near the OEC/ONF-biomatrix complexes in the graft/lesion sites, a histological Hoechst staining was performed after sacrificing the animals to investigate whether host cells migrated into the transplanted complexes. Here, it became obvious that many host cells invaded the biomatrix complexes (Fig. 1E). Additional immuno staining furthermore showed that a subpopulation of these invading cells was $\mathrm{p}^{75}$-NGF-receptor-positive, suggesting them to be invading Schwann cells (Fig. 1F). The identity of the rest of the invading cells remains elusive.

Fig. 1. Transplanted Hoechst-prelabeled OEC/ONF and infiltrating host cells into the lesion site. One day prior to transplantation, OEC/ONF were prelabeled with Hoechst 33342. (A) S100 $\beta$ immunostained and Hoechstprelabeled OEC/ONF fixed at the day of transplantation (examples of S100 $\beta$ immunostaining (arrowheads) and Hoechst staining (arrows) are indicated. (B, C) Prelabeled OEC/ONF present in a limited area (around the injection site) rostral to the injury site at 9 weeks after injury/transplantation. The dotted line in panel B delineates the spinal cord; the arrow indicates the location of the lesion site; d, dorsal; v, ventral; r, rostral; c, caudal; panel $\mathrm{C}$ is an enlargement of the box shown in panel B. (D) Transplanted Hoechst-prelabeled cells (blue) in the injection area; note that a proportion of the cells are immunoreactive for $\mathrm{p}^{75}$-NGF receptor (red). Arrows indicate a few Hoechst-prelabeled cells with $\mathrm{p}^{75}$-NGF receptor immunoreactivity. (E) Hoechst staining of the OEC/ONF-biomatrix complex within the lesion site; all these Hoechst-positive cells are infiltrating host cells (note that the Hoechst staining does not reflect any prelabeled cells, but only cells that are labeled after sacrificing the animals). (F) Identification of infiltrating Hoechst-stained (blue) host cells within the biomatrix complex; a subpopulation of the cells is immunoreactive for $\mathrm{p}^{75}$-NGF receptor (red). Scale bars in A-C, E, and F are $200 \mu \mathrm{m}$; scale bar in panel D is $100 \mu \mathrm{m}$.
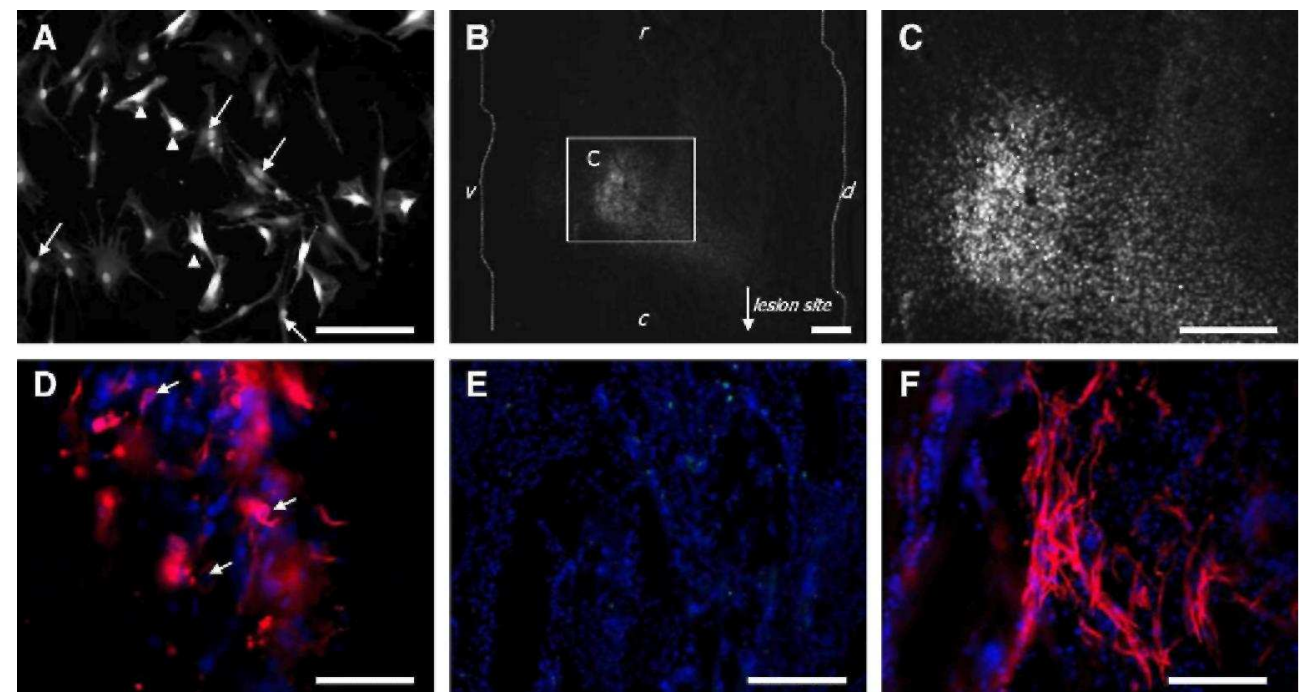

Fig. 2. Transplantation of OEC/ONF-biomatrix complexes in dorsal hemisection injury sites. After a laminectomy of the low-thoracic spinal cord (A), a 2-mm-long dorsal hemisection gap was made (B). Hereafter, OEC/ONF suspensions were injected at $1 \mathrm{~mm}$ rostral and $1 \mathrm{~mm}$ caudal to the injury site (approximate injection sites are indicated by arrowheads) and an aligned OEC/ONF-biomatrix complex was inserted into the lesion gap (C). This complex was stabilized by a Dura Film covering (not shown).
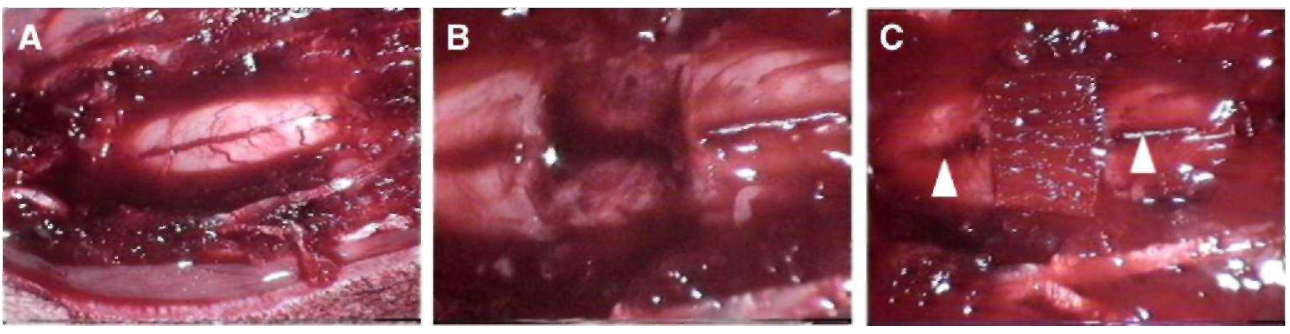
Fig. 3. Neurofilament IR fiber ingrowth into the graft/lesion site. The graft/lesion site was delineated as indicated in the schematic representation in panel A; the asterisk in panel A represents the approximate location of the section shown in panel B. GFAP immunostaining was used to identify the border of the graft/lesion site. Numerous neurofilament IR fibers were observed to pass the GFAP-IR tissue (arrow in panel B) and grow into the graft/lesion site (B). In control animals, neurofilament IR fibers also passed the GFAP-IR tissue but then stayed at the outer circumferences of the lesion gap. Quantification of neurofilament IR fiber ingrowth into the graft/lesion site showed a 10-fold increase in transplanted versus control animals (C). Serotonin immunohistochemistry shows that serotonergic fibers approach the graft/lesion site (indicated with an asterisk) (D). Arrowheads in panel B indicate parts of the PLA/PLA- $b$-PEO biomatrix material; R, rostral; C, caudal; *P $<0.05$.

A
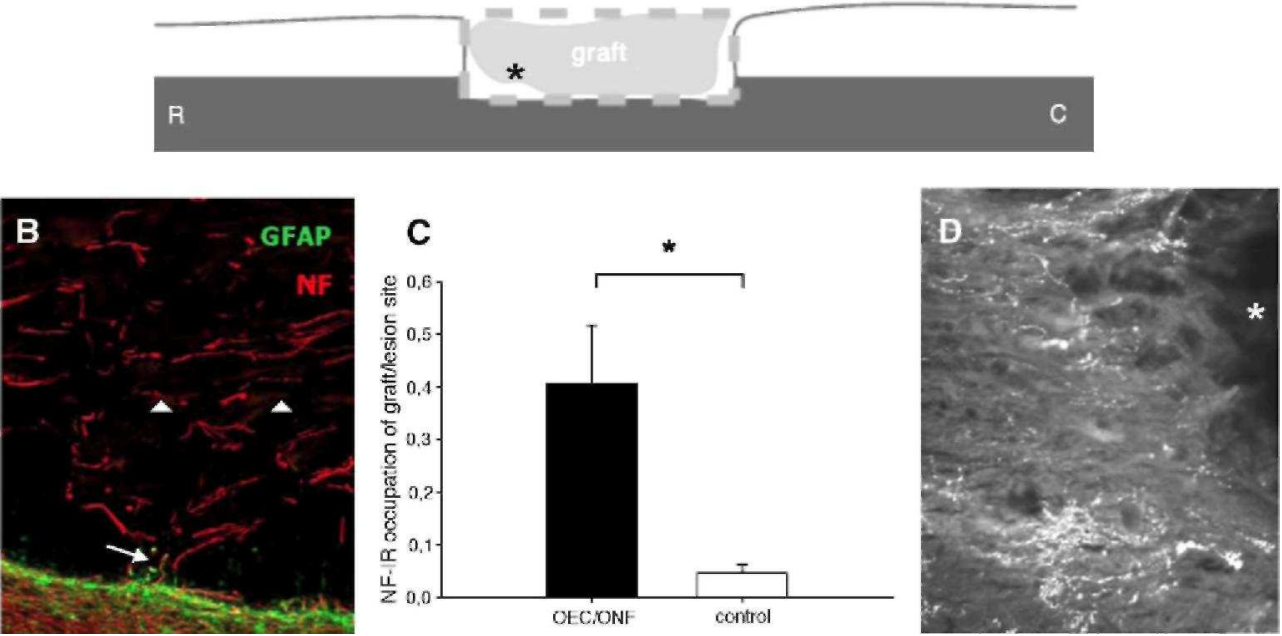

Ingrowth of axons into the graft/lesion site

Before focusing on axon regrowth per se, we were interested to see whether axons penetrated the transplanted OEC/ONF-biomatrix complex, and we therefore performed a general neurofilament (NF) staining. In control animals, there was occasionally an NF-positive fiber passing the GFAP-positive scar. However, NF-positive fiber growth into the lesion site was restricted to the outer circumferences of the lesion (i.e. at the border of the lesion gap). In sharp contrast to this, numerous NF-positive fibers were observed to grow into the OEC/ONFbiomatrix complexes of transplanted animals (Fig. 3B), but also into the DuraFilm covering the grafts. Next, a quantitative analysis was performed. Because the complex was designed to completely fill up the lesion site, we analyzed the ingrowth of NF-positive fibers ("NF immunoreactivity occupation") in the complete lesion gap (irrespective of the degree in which the complex filled up this lesion gap), using the GFAP-positive scar to delineate the graft/lesion site (Fig. 3 A). The size of the graft/ lesion site, as demarcated by the GFAP-IR boundary, did not differ between OEC/ONF-transplanted animals and control animals (data not shown). The occupation of the graft/lesion site by NF-positive fibers was $0.41 \pm 0.11 \%$ in transplanted animals versus $0.05 \pm$ $0.02 \%$ in control animals (Fig. $3 \mathrm{C}, P<0.05$ ). Although serotonin immuno staining showed nicely stained serotonergic fibers in the intact spinal tissue (Fig. 3D), the graft/ lesion site was completely devoid of serotoninimmunoreactive fibers (data not shown).

\section{CS axon response}

BDA labeling of the CS tract was successful in nine out of ten transplanted animals and in seven out of eight control animals. BDA-labeled CS axons were clearly observed to approach the graft/lesion site (Figs. 4A, C, D). Most of these axons stopped either before or at the host/graft interface (Figs. 4A, C, D). No BDA-labeled CS axons were observed to penetrate the graft in any of the animals. Occasionally, a BDA-labeled CS axon was observed underneath the graft/lesion site. In one or two of the transplanted and control animals, this was also the case caudal to the lesion site (Fig. 4E). The anterograde labeling efficiency largely varied among the animals: BDA immunoreactivity at the rostral reference was $12.3 \%$ at maximum and $0.15 \%$ at minimum. No statistically significant differences in BDA-labeled CS axons were present between the two groups of animals. These values were used as the $100 \%$ reference values with respect to the BDA immunoreactivity occupation measures rostral, 
underneath, and caudal to the lesion. Quantification of BDA immunoreactivity occupation at the four different locations and statistical testing using ANOVAR with Bonferroni post hoc showed that there was an overall difference in BDA immunoreactivity over the respective locations (Location: $F_{1,14}=504.85, P<0.001$ ). Fig. 4B shows a reduction in BDA immunoreactivity (1) directly rostral to the lesion site, (2) underneath the lesion site, and (3) caudal to the lesion site as compared to the rostral reference in both transplanted and control animals. In addition, an overall group difference was found (Group: $\left.F_{1,14}=12.09, P<0.01\right)$. A significantly $(P<0.01)$ higher BDA immunoreactivity was detected directly rostral to the lesion/graft site in transplanted animals $(65.0 \pm$ $12.8 \%)$ as compared to control animals $(13.1 \pm 3.9 \%)$.

We reasoned that the higher BDA immunoreactivity directly rostral to the injury site could reflect a sprouting response by the injured CS axons, a reduction in CS axon die-back, or a combination of these two options. In order to test whether the CS axons approaching the host/graft interface were sprouting axons, a BDA/GAP43 double staining was performed. All the nine transplanted and seven control animals with a good BDA labeling of the CS tract staining were analyzed for GAP43-positive BDA-immunoreactive axons. In none of the transplanted or control animals did we observe such axons (Fig. 4D), suggesting that the transplantation effect on the injured $\mathrm{CS}$ axons rostral to the lesion site most likely involves a reduced die-back.

\section{Functional outcome}

\section{BBB locomotor rating scale}

The spinal cord injury resulted in functional impairment of the animals. At the first day after injury, the majority of animals included into this study were not able to display plantar placement of their hind paws, although there was extensive movements of the hip, the knee, and the ankle. In the first week after injury, all the animals had plantar placement of the hind paws, many with weight support. At 3 weeks after injury, all the animals showed consistent weight supported plantar stepping (BBB score 11), and some of them displayed occasional coordination between the forelimbs and the hind limbs (BBB score 12).

Throughout the 9 weeks post-injury, the animals were followed in the BBB locomotor test on a weekly basis. No difference was in BBB scores observed between transplanted and control animals at any time point (Fig. 5A). However, most of the animals in both groups remained at a BBB score of 11 from the third week to the ninth week. A BBB score of 11 reflects frequent to consistent weight supported plantar stepping without coordination (Basso et al., 1995).

\section{CatWalk-based BBB scale}

In the CatWalk gait analysis, the RI can be regarded as an objective measure of coordination. When the CatWalk-based coordination data are integrated into the BBB scores at 3, 6, and 9 weeks after injury, the BBB scores were dramatically increased (Fig. 5B). Transplanted animals increased their BBB scores from 11.4 (DP021), 11.8 (DP042), and 11.4 (DP063) to 15.2 (DP021), 15.3 (DP042), and 15.8 (DP063), all statistically significant using a Student's $t$ test $(P<0.01)$. Furthermore, control animals increased their BBB scores upon integration of the CatWalk-based coordination data: from 11.1 (DP021), 11.8 (DP042), and 11.6 (DP063) to 15.4 (DP021), 14.6 (DP042), and 13.6 (DP063); except for DP063, all these time points are statistically significant using a Student's $t$ test $(P<0.01)$. Since the BBB scores increased to a similar extent in both the transplanted and control animals, no significant difference could be noted between the two groups upon integration of the CatWalk-based coordination data into the BBB scores at any time point (Fig. 5B).

\section{CatWalk gait analysis}

\section{Duration of walkway crossing}

With respect to the CatWalk gait analysis, it is important to know that the duration of walkway crossing can have an influence on the locomotion of the animals (Koopmans et al., 2005). Therefore, it is needed to have identical durations of walkway crossing in both animal groups when comparing the various locomotor parameters. Preoperative training on the CatWalk resulted in rapid runway crossings of $1.39 \pm 0.07 \mathrm{~s}$ and $1.47 \pm 0.06 \mathrm{~s}$ in transplanted and control animals, respectively.

Three weeks after injury, the runway crossing increased significantly to $2.11 \pm 0.12 \mathrm{~s}$ and $2.45 \pm 0.25 \mathrm{~s}$ in transplanted and control animals, respectively. At the 6 and 9 weeks post-injury time points, there was no further increase in runway crossing in both animal groups. A statistically significant increase in crossing time between 
the preoperative and post-injury time points in both animal groups was noted (Time: $F_{3,39}=7.37, P<0.001$; and $F_{3,31}=4.71, P<0.01 ; 1$-way ANOVA/Bonferroni post hoc over four time points for transplanted and control animals, respectively). However, more importantly, no statistically significant difference in crossing time was found between the two animal groups at any of the four time points, and, thus, a legitimate comparison of data between the two groups can be made at any given time point.

Fig. 4. BDA-IR corticospinal axon response to OEC/ONF intervention. BDA-IR corticospinal axons were observed to approach the graft/lesion site, but only very few grew underneath the lesion and into the caudal host spinal cord. No labeled corticospinal axons penetrated the graft/lesion site. (A) Sagittal spinal cord section of an OEC/ONF-transplanted animal. The dotted line represents the delineation of the spinal cord; the OEC/ONFbiomatrix complex is visible within the lesion site. The four boxes represent the boxes used in the quantitative analysis. (B) A significantly higher BDA-IR is present directly rostral to the injury site (box 2) in transplanted animals versus control animals. (C) BDA/GFAP-stained sagittal section of an OEC/ONF-transplanted animal; many BDA-labeled corticospinal axons stop rostral to the graft/lesion site; asterisk in panel C indicates the graft/lesion site. (D, E) BDA/GAP43-stained sagittal sections of OEC/ONF-transplanted animals showing that labeled corticospinal axons rostral to the graft/lesion site are not GAP43 IR (D), but numerous GAP43 IR fibers are present within the graft/lesion site (note that panel $\mathrm{E}$ is in the middle of the graft/lesion site) (E); asterisk in panel D indicates the graft/lesion site. (F) BDA-labeled corticospinal axon (arrow) present within the caudal gray matter of an OEC/ONF-transplanted animal; these caudal fibers were only noted in one or two transplanted and control animals. Scale bars are $100 \mu \mathrm{m}$.

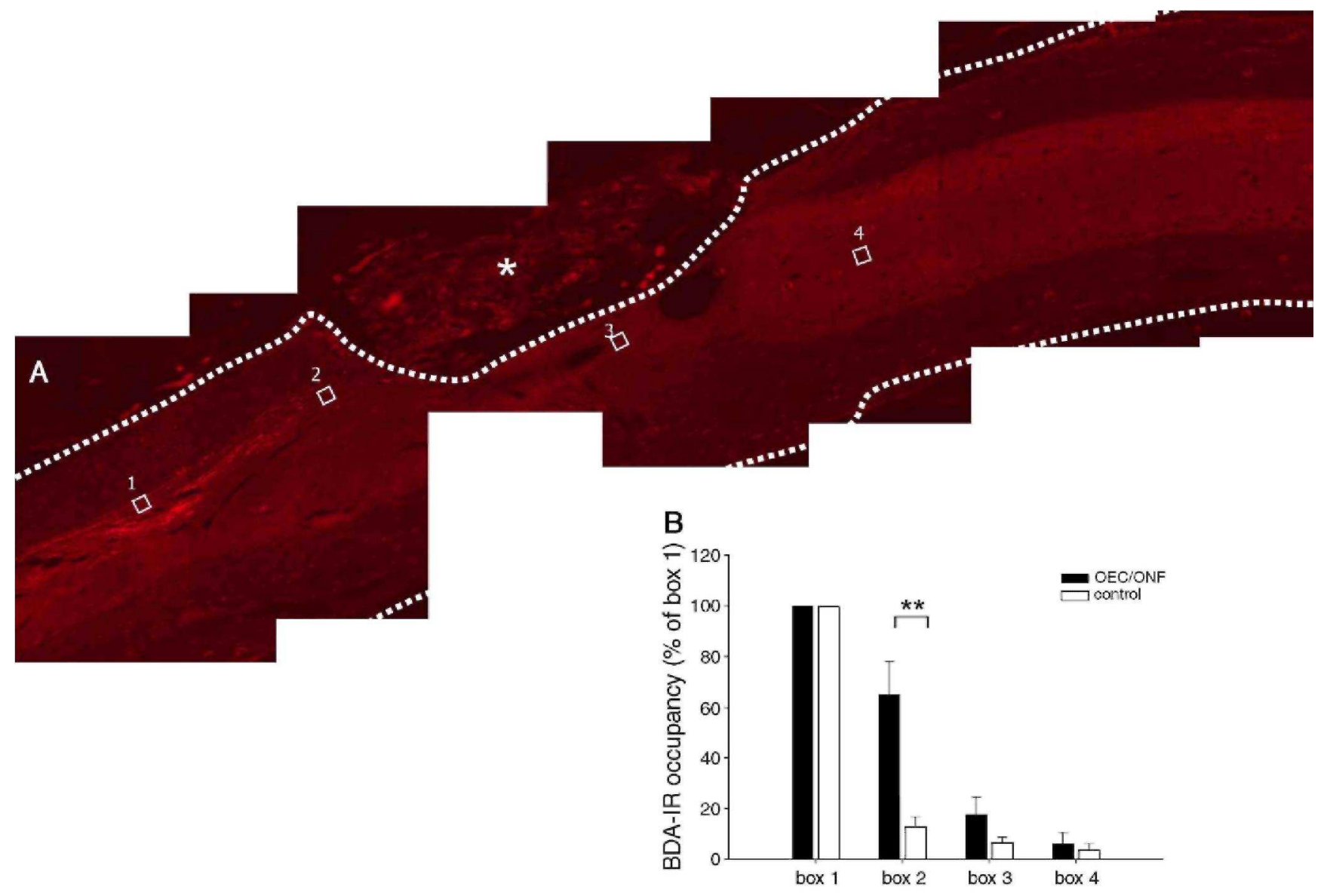




\section{Fig 4 (continued)}
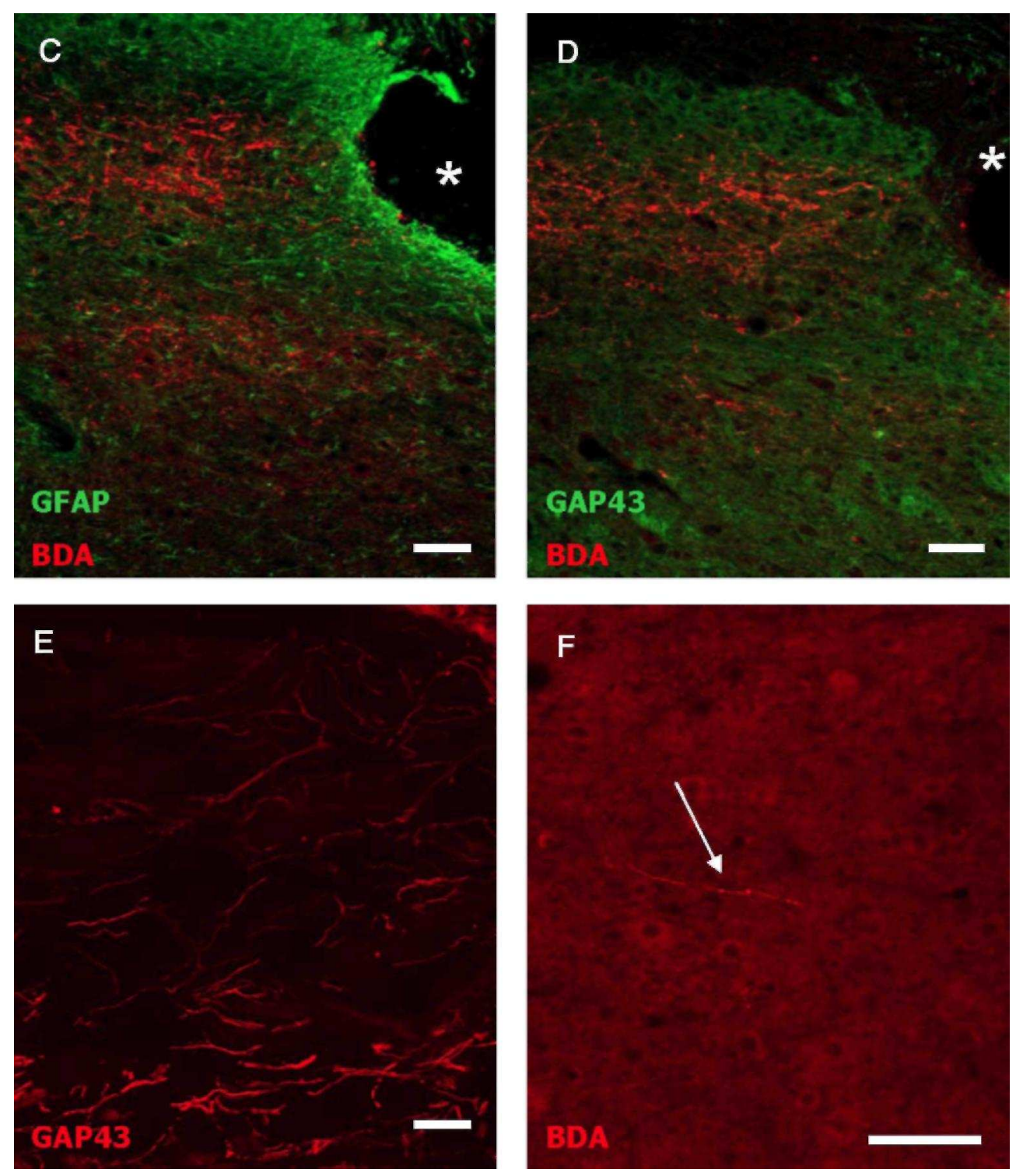

\section{Stride length of hind limbs}

The stride length of the hind limbs is the distance between the print of one hind paw in one step cycle to the print of the same hind paw in the next step cycle. Before injury, the stride length of transplanted and control animals was $148.1 \pm 2.8 \mathrm{~mm}$ and $140.2 \pm 2.6 \mathrm{~mm}$, respectively (n.s.; Fig. 5C). Over the four different time points, there was a statistically significant decrease (Time: $F_{1,16}=18.14, P<0.01$ ). Furthermore, an overall significant difference was observed between the two groups (Group: $F_{1,16}=6.11, P<0.05$ ). At the last time point in this study (i.e. 9 weeks post-injury), Student's $t$ testing showed that the stride length of the hind limbs in transplanted animals was significantly higher than in control animals $(134.5 \pm 3.1 \mathrm{~mm}$ and $122.5 \pm 3.3 \mathrm{~mm}$, respectively; $P$ < 0.05; Fig. 5C).

\section{Swing speed of hind limbs}

This parameter combines the distance (stride length) that the hind paw crosses within one step cycle and the time (swing duration) it takes. Before injury, the swing speed of the hind limbs was $1.20 \pm 0.05 \mathrm{~m} / \mathrm{s}$ and $1.15 \pm 0.02$ $\mathrm{m} / \mathrm{s}$ for transplanted and control animals, respectively (n.s.). Overall, a tendency was observed for a difference between the two animal groups (Group: $F_{1,16}=4.34, P=0.054$ ). Over all time points, there was a significant change in swing speed (Time: $F_{1,16}=9.47, P<0.01$ ). Student's $t$ tests showed that the swing speed of the hind 
limbs was significantly higher in transplanted animal as compared to control animals at the end of the experiment (i.e. 9 weeks after injury) (Fig. 5D; $P<0.05$ ). Although inter-group changes were found for both stride length of the hind limbs and swing speed of the hind limbs, there was no change in swing duration between the two animal groups (data not shown).

Fig. 5. Behavioral effects of OEC/ONF intervention. The acute OEC/ONF transplantation did not have an effect on gross locomotion as reflected by the outcome of the BBB locomotor scale (A) and the CatWalk-based BBB scale (B). Fine locomotor parameters as assessed in the CatWalk gait analysis showed a significant difference after acute OEC/ONF transplantation at 9 weeks after injury: stride length and swing speed of the hind limbs were significantly improved in transplanted versus control animals $(\mathrm{C}, \mathrm{D}) . * P<0.05$.

A

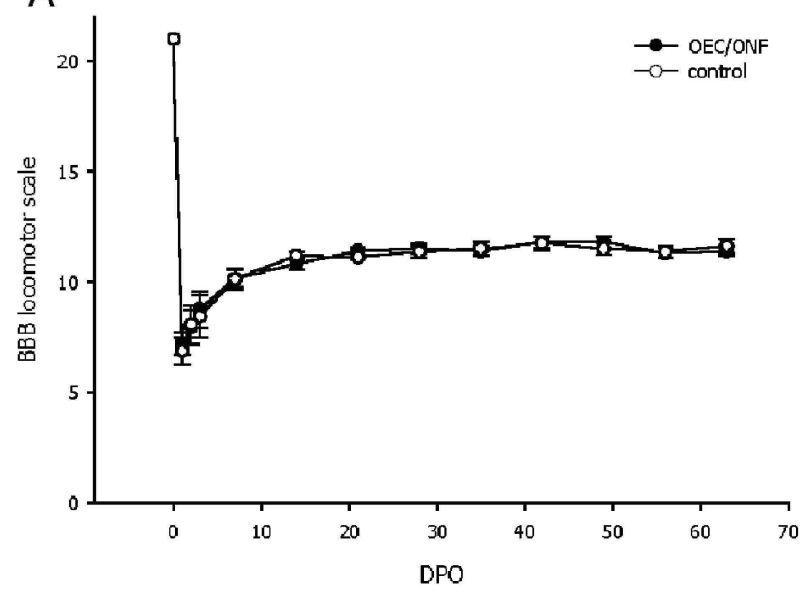

C

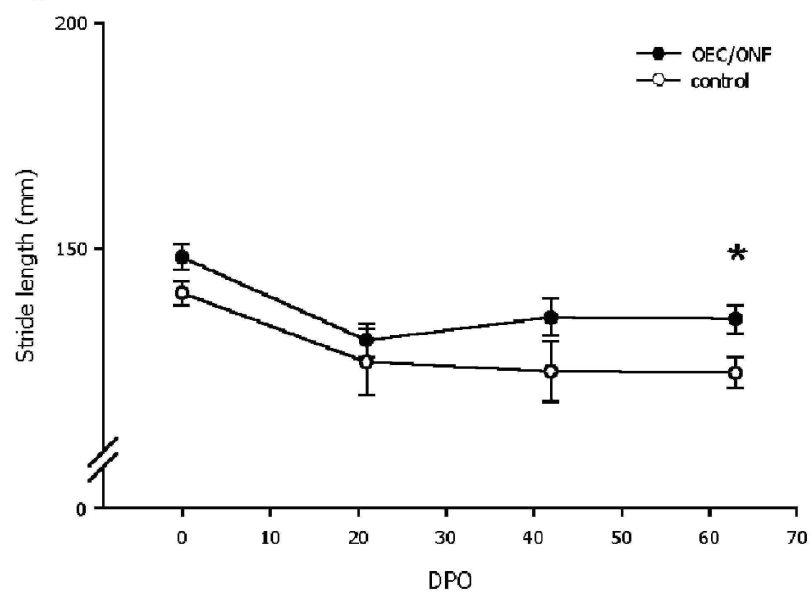

B

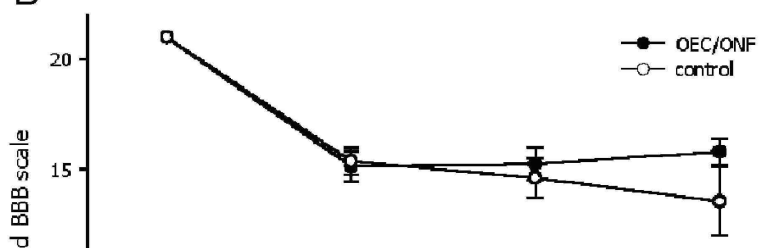

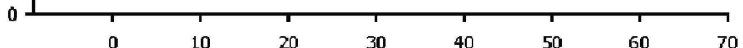
DPO

D

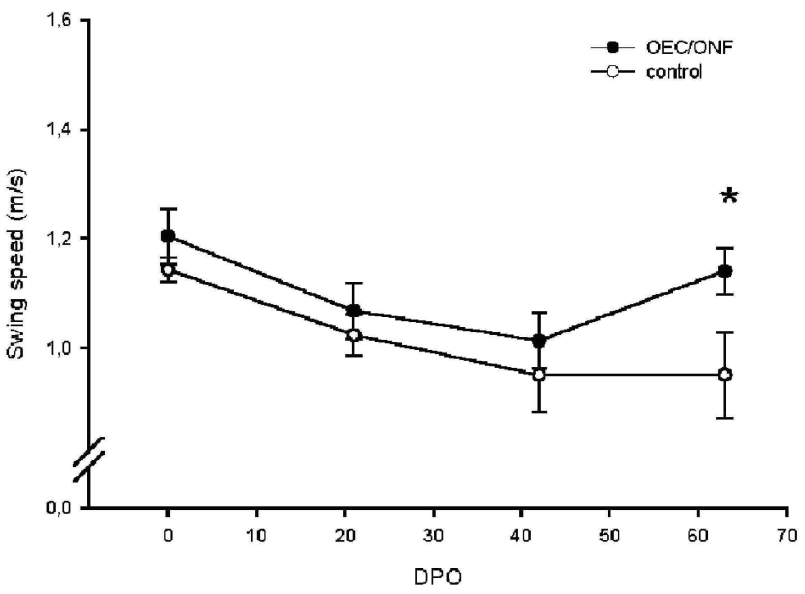

\section{Discussion}

We studied the effect of a multifactorial acute transplantation strategy on axon regrowth across large spinal lesion gaps. This strategy designed to create an OEC/ONF continuum in the injured spinal cord involved two aspects: an aligned OEC/ONF-biomatrix complex to bridge a 2-mm dorsal hemisection lesion site and additional OEC/ONF cell injections at $1 \mathrm{~mm}$ rostral and $1 \mathrm{~mm}$ caudal to the injury site (Fig. 2). We show that the transplanted OEC/ONF survived at the injection areas, but not on the biomatrices within the lesion gaps. Furthermore, the surviving OEC/ONF in the injection areas did not migrate from these areas. Hence, an OEC/ONF continuum was not formed. Nevertheless, we demonstrated that our transplantation strategy stimulated numerous axons to grow into the OEC/ONF-biomatrix complex. None of these axons was of CS or raphespinal origin. Although BDA-labeled CS axons did not enter the graft in any of the animals, the transplantation strategy resulted in a clear increase of injured BDA-labeled CS axons directly rostral to the injury 
site. This response of injured BDA-labeled CS axons may reflect a reduced die-back of the axons as a result of the transplantation intervention since none of these CS axons was GAP43/B50-positive. In addition, the transplantation strategy used stimulated a recovery in stride length and swing speed of the hind limbs.

\section{OEC vs. OEC/ONF}

Currently, there is an ongoing discussion about the optimal composition of olfactory ensheathing cell cultures to be used for stimulating regeneration of axons severed by an experimental spinal cord injury (Barnett and Chang, 2004). Acute transplantation of mixed OEC/ONF cultures has stimulated regrowth of injured CS axons across relatively small spinal lesion gaps (Li et al., 1997, 1998). However, when purified OEC cultures were transplanted into complete or incomplete transection sites, injured CS axons were also observed to cross the relatively small lesion gaps (Ramon-Cueto et al., 2000; Nash et al., 2002). Strikingly, when regrowing across these small lesion gaps, injured CS axons were reported to preferentially use the spinal cord surface in the presence of meningeal fibroblasts (Ramon-Cueto et al., 2000). This suggests a cooperation of the transplanted OEC with fibroblasts. In contrast to small spinal lesion gaps, large spinal lesion gaps require long-distance axon regrowth. Acute transplantation of purified OEC cultures has stimulated very few injured CS axons to re grow around large spinal lesion gaps (Chuah et al., 2004). Recently, it was claimed that OEC and ONF are involved in long-distance olfactory axon regeneration ( $\mathrm{Li}$ et al., 2005). However, in our multifactorial transplantation strategy in the spinal cord, transplanted OEC/ ONF were not able to stimulate long-distance regrowth of injured CS axons.

\section{Multifactorial transplantation strategy and regrowth of injured CS axons across large spinal gaps}

Although clinically relevant, repair of large spinal lesion gaps has been quite unexplored. The long-distance axon regrowth, required in large spinal lesion gaps, may be influence by at least three issues. First, a continuum of growth-promoting cells within the spinal cord. Injured CS axons were found to regrow into grafts composed of immature astrocytes in a collagen matrix but did not re-enter the caudal host spinal cord (Joosten et al., 2004). It was suggested that this lack of CS axon re-entry into the host tissue was influenced by the absence of transplanted cells in the host tissue. Second, the alignment of cells transplanted into the large lesion gaps. A specific organization of astrocytes has been proposed to be of utmost importance for the correct long-distance outgrowth of the CS tract during development (Joosten and Gribnau, 1989). Furthermore, a clear orientation of transplanted OEC has stimulated directional host axon growth from the thalamus to the hippocampus (PerezBouza et al., 1998). Third, since OEC and ONF stimulate long-distance olfactory axon regeneration (Li et al., 2005), the use of these mixed cultures may also be beneficial to stimulate long-distance regrowth of axons severed by an SCI. In our multifactorial transplantation strategy, all these three issues were addressed. First, OEC/ONF were transplanted both into the 2-mm dorsal hemisection lesion gap (cultured on biomatrices) and into the rostral and caudal host tissue. Putative migration of OEC/ONF within the injured spinal cord, as described previously (Li et al., 1998), may then create an OEC/ONF continuum. Second, aligned OEC/ONFbiomatrix complexes, which are known to stimulate directional neurite outgrowth in vitro (Deumens et al., 2004), were transplanted into the lesion gaps. Third, mixed OEC/ONF cultures were used.

Our multifactorial transplantation intervention has rendered two main histological outcomes. First, there was an enhanced presence of injured CS axons directly rostral to the spinal lesion gap (Fig. 4). Second, numerous axon penetrated the OEC/ ONF-biomatrix complexes in the spinal lesion gaps (Fig. 3). Interestingly, these axons seemed to have an aligned phenotype.

The enhanced presence of CS axons directly rostral to the lesion gap may reflect a reduced die-back of injured CS axons because the injured CS axons were immunonegative for GAP43/B50. Die-back of injured CS axons normally starts directly after injury and renders the majority of CS axons to be located between 1 and $2.5 \mathrm{~mm}$ rostral to the injury site at 8-10 weeks after trauma (Pallini et al., 1988; von Meyenburg et al., 1998; Oudega et al., 1999). Our results are in sharp contrast to those obtained after transplanting mixed OEC/ONF cultures in small spinal lesion gaps, thereby stimulating regrowth of CS axons through the spinal lesion sites and into the caudal spinal tissue up to $1 \mathrm{~cm}$ ( $\mathrm{Li}$ et al., 1997, 1998). We propose three possibilities involved in the contrasting results. First, OEC/ONF transplants readily survived in small lesion gaps (Li et al., 1997, 1998, 2003). In our study, no Hoechst-prelabeled OEC/ONF were observed on the OEC/ONF-biomatrix complexes transplanted into the large lesion gap (see below). Second, the lesion model of Li and co-workers strongly differed from ours. The level of the lesion differed, i.e. high cervical vs. low thoracic. The capacity of an injured axon to regrow is decreased with an increasing distance between the injury and the soma of the injured neuron (Richardson et al., 1984). Next to this, the extent of the lesion differed, i.e. defined lesion of the CS tract vs. 2-mm-wide dorsal hemisection injury. Although injured CS axons may regrow across small spinal lesion gaps after one-way 
approaches such as simply injecting OEC/ONF suspensions (Li et al., 1998), long distance of injured CS axons across large lesion gaps may require additional interventions, such as the use of growth-promoting bridges within these large lesion gaps.

Although such bridges may consist of growth-promoting cells and biodegradable matrices, our study clearly shows some disadvantages of the specific combination of OEC/ONF with poly(D,L)-lactide matrices like a lack of OEC/ONF survival on the matrices within the lesion site and a poor integration of the complexes into the host spinal tissue (see also below). Third, CS axon regrowth across a 2-mm-long lesion gap and into the caudal host tissue may require more than the 9 weeks. A 9-week period has been shown sufficient for CS axon regrowth across relatively small lesions (Li et al., 1998). However, our data, showing CS axons present almost exclusively rostral to the injury site, suggest that the time after injury is not the determining factor in the CS axon regrowth response. Although inter-group differences in CS axon regrowth were only found directly rostral to the injury site, in both OEC/ONF-transplanted animals and untreated control animals, a few CS axons were observed in the gray matter underneath the lesion site and within the gray matter of the caudal spinal tissue. In line with this, others found a similar CS axon regrowth response after OEC transplantation into large lesion gaps associated with thoracic dorsal column transection (Chuah et al., 2004).

Concerning NF-positive axon growth into the lesion site, we previously showed a spontaneous response of endogenous tissue repair after dorsal hemisection injury of the thoracic spinal cord of the rat (Brook et al., 2000). Then, NF-positive fibers were found to spontaneously regrow into small dorsal hemisection lesion gaps, which were infiltrated by numerous host cells expressing the $\mathrm{p}^{75}$-NGF receptor. In the present study, such a spontaneous response of endogenous tissue repair did not occur since the extent of the injury was much larger by taking out the dorsal half of the spinal cord over a distance of $2 \mathrm{~mm}$. Control animals, which did not receive a transplant, obviously had only a minor penetration of NF-positive axons into the lesion site. This may be explained by the lack of host cell infiltration into the lesion site. On the contrary, numerous NF-positive axons penetrated the graft/lesion site of OEC/ONF-transplanted animals. Noticeably, although OEC/ONF were only present on the surface area of the OEC/ONF-biomatrix complexes upon transplantation, NF-positive axons were observed throughout the complex. Many host cells, presumably Schwann cells (as we observed immunoreactivity for the $\mathrm{p}^{75}$-NGF receptor) and fibroblasts (Bunge et al., 1997; Pasterkamp et al., 1999), were found to invade the biomatrix complexes (possibly by growing within the pores of the biomatrix). These invading host cells may account for the stimulation of NF ingrowth into the graft. All the NF-positive axons in the graft/ lesion site were GAP43/B50-positive, confirming their regrow-ing/sprouting nature. Although the origin of these ingrowing axons is yet unclear, a CS and raphespinal origin could be excluded. Obviously, these ingrowing axons may have a peripheral origin (e.g. CGRP immunoreactive axons), as has been described previously in a less severe injury model (Brook et al., 2000). Quantitatively, OEC/ONF-transplanted animals showed a 10-fold increase in NF-positive axon growth into the graft/lesion site as compared to control animals. Since our transplantation strategy is multifactorial, an argument may arise around the exact mechanisms stimulating neurofilament-positive axon growth into the transplanted OEC/ONF-biomatrix complexes: is it the transplanted OEC/ONF or the biomatrix? Pilot experiments by our group, using a similar quantification procedure, showed that there was no difference between the ingrowth of NF axons into OEC/ONF-biomatrix grafts or biomatrix grafts without OEC/ONF. Hence, the biomatrix itself may already be sufficient to stimulate NF axon ingrowth into the graft/lesion site. This effect is presumably mediated by the host cells invading the biomatrix after transplantation.

\section{Survival and migration of transplanted OEC/ONF}

Although no Hoechst-prelabeled-positive cells could be detected within the spinal lesion gap, many prelabeled cells were observed within the injection areas (Fig. 1). A minority of these cells expressed the $\mathrm{p}^{75}$-NGF receptor, which was expected because of the presence of this receptor on the OEC in mixed OEC/ONF cultures (Li et al., 1998; Deumens et al., 2004). Although Hoechst can leak out of transplanted cells and stain host cells (Iwashita et al., 2000), the condensed presence of Hoechst-positive cells and the $\mathrm{p}^{75}$-NGF receptor immunoreactivity in a subpopulation of Hoechst cells suggests that the Hoechst-positive cells within the injection areas are transplanted OEC/ONF that survived. Whereas the injected OEC/ONF survived transplantation into the injection areas, they did not migrate within the injured spinal cord. There are some essential differences between our study lacking OEC/ ONF migration and other studies showing migration of olfactory glia after injection into the injured spinal cord (Li et al., 1998; Ramon-Cueto et al., 2000), including the specific olfactory glia culture and the injection procedures. However, the influence of any of those factors on migration of the transplanted olfactory glia remains to be determined. The lack of survival of transplanted OEC/ONF on the biomatrices and the lack of migration of injected OEC/ONF may have impeded CS axon regrowth because no OEC/ONF continuum was created. Pathological processes, like hemorrhage and inflammation, strongly affect the injured spinal cord 
environment (Dusart and Schwab, 1994). The concentration of toxic substances released from such processes may be higher in spinal lesions which are larger (Wagner et al., 1978). Obviously, this may have influenced the migration of the transplanted OEC/ONF within the host spinal tissue. The lack of OEC/ONF migration within the host spinal tissue was also reported by others who transplanted purified OEC cultures into the host spinal tissue close to large spinal lesion gaps (Chuah et al., 2004). Hence, it is advisable to use multiple cell injections to create a continuum of these cells with spinal cords with large lesion gaps.

Next to this, the lack of OEC/ONF survival within the large spinal lesion gap is in contrast with previous findings (Sasaki et al., 2004), and a combination of several causes may be involved. First, the putative high concentration of toxic substances released by pathological processes may have affected the survival of OEC/ONF-transplanted into these large lesion gaps. Since the OEC/ONF were cultured on the biomatrix surface facing the injured spinal tissue, the toxic substances are directly available to these transplanted OEC/ ONF. Indeed, previous studies have demonstrated the survival of transplanted cells in identical large lesion gaps when the cells were embedded in collagen type I matrices and were thereby relatively protected from the injured spinal cord environment (Joosten et al., 2004). Second, the acutely injured spinal cord has been shown to have a negative effect on OEC proliferation and increase OEC apoptosis as compared to either uninjured or chronically injured spinal cord tissue (Woodhouse et al., 2005). Third, optimal numbers of OEC/ONF have been used to create aligned OEC/ONF-biomatrix complexes during 4 days in vitro (Deumens et al., 2004). These numbers are relatively low and may have negatively affected the survival of the transplanted OEC/ONF within the spinal lesion sites upon transplantation of these complexes. Fourth, there are also some differences between our study set-up and that of Sasaki and colleagues which could affect OEC survival, including a difference in lesion type, studied time points after injury, and cell preparation protocols. Finally, the specific use of poly(D,L)-lactide matrices to transplant OEC/ONF into the large spinal lesion gaps may have negatively affected OEC/ONF survival. Degradation of poly(D,L)-lactides is for example associated with a lowering of the $\mathrm{pH}$ (Mainil-Varlet et al., 1997), which may impair OEC/ ONF survival. Hence, biomaterials other than poly(D,L)-lactides may be tested in combination with OEC/ONF for their effects on long-distance CS axon regrowth across large spinal lesion gaps.

\section{Functional recovery}

The multifactorial transplantation strategy stimulated modest recovery of fine locomotor parameters (Fig. 5). The (incomplete) recovery of locomotor functions (swing speed and stride length of the hind limb) was observed at 9 weeks after injury, which may reflect an involvement of axonal regrowth. With the current knowledge, directly linking regrowth of individual axon tracts and functional recovery is impossible (Deumens et al., 2005). Nevertheless, an improvement in stride length as demonstrated in this study has previously been reported together with enhanced regrowth of injured CS axons across a thoracic dorsal hemisection lesion site and back into the caudal host tissue (Bregman et al., 1995). Furthermore, others have reported a relation between the CS tract and stride length of the hind limbs (Starkey et al., 2004). In our study, no relation between regrowth of the injured CS tract axons and recovery of stride length could be determined. Presumably, the effects on injured CS axons are paralleled by responses of other axon tracts descending from the brain that are more likely to be linked with stride length. Besides this, formation of new intraspinal relay circuits as described by others (Bareyre et al., 2004) cannot be excluded.

The effect of our intervention on locomotion is restricted to only a small subset of locomotor parameters. This is very likely directly related to the spinal cord injury model that we used. We are the first to evaluate behavioral impairments after dorsal hemisection injury quantitatively in such a highly detailed, objective, and sensitive manner using the CatWalk gait analysis. On a behavioral level, the CatWalk showed that the control animals in our study were not as severely impaired as expected. The majority of locomotor parameters assessed in the CatWalk remained unaffected. Among these unaffected parameters is the regularity index (RI), which is strongly involved in coordination. Implementation of RI as an objective parameter for coordination into the BBB test resulted in a significant increase of the BBB score of both transplanted and control animals. In a previous study of our group using a spinal cord contusion injury, RI implementation into the BBB showed a strong enhancement in the sensitivity of the BBB (Koopmans et al., 2005). Here, a significant difference in BBB scores was demonstrated in enriched housed animals as compared to normally housed animals, only upon implementation of the RI in the BBB (Koopmans et al., 2005). When the RI was implemented into the BBB in the present study, the BBB scores of transplanted and control animals increased to a similar extent, and, thus, no inter-group difference could be observed. This more sensitive and objective behavioral analysis again emphasizes the rather limited behavioral impairment after dorsal hemisection injury, leaving open an only very small window for functional recovery. 


\section{Conclusions}

Our multifactorial transplantation strategy was inefficient in stimulating regrowth of injured CS axons across large spinal lesion gaps. However, an enhanced presence of injured CS axons was observed directly rostral to the lesion gap, which was paralleled by the penetration of many axons into the graft and by modest locomotor recovery. Future studies may focus on optimalization of the transplantation strategy and thereby address issues, such as (1) the limited migration of transplanted OEC/ONF within the host tissue of spinal cords with large lesion gaps and (2) the problems with OEC/ ONF survival on poly(D,L)-lactide matrices within large lesion gaps. Furthermore, we recommend the combined use of detailed and sensitive behavioral tests such as the CatWalk gait analysis and a spinal injury model with a higher degree of behavioral impairment in order to have a much wider behavioral window for the determination of therapeutic effects.

\section{Acknowledgments}

The authors thank Dr. Y. Li and Prof. Dr. G. Raisman for extending their knowledge on OEC/ONF culturing to our laboratory and Marijke Lemmens and Dr. Jos Prickaerts for their assistance with the immunohistochemical stainings and expertise regarding picture processing, respectively. The authors are also grateful to Lou Kirkels (IDEE, Maastricht) for his expertise with respect to the injection procedures. The RT97 monoclonal antibody, developed by Dr. J. Wood, was obtained from the Developmental Studies Hybridoma Bank developed under the auspices of the NICHD and maintained by The University of Iowa, Department of Biological Sciences, Iowa City, IA 52242, USA. This work was funded by the International Spinal Research Trust (ISRT; STR057 to E.A.J.J.)

\section{References}

Bareyre, F.M., Kerschensteiner, M., Raineteau, O., Mettenleiter, T.C., Weinmann, O., Schwab, M.E., 2004. The injured spinal cord spontaneously forms a new intraspinal circuit in adult rats. Nat. Neurosci. 7, 269-277.

Barnett, S.C., Chang, L., 2004. Olfactory ensheathing cells and CNS repair: going solo or in need of a friend? Trends Neurosci. 27, 54-60.

Basso, D.M., Beattie, M.S., Bresnahan, J.C., 1995. A sensitive and reliable locomotor rating scale for open field testing in rats. J. Neurotrauma 12, 1-21.

Bregman, B.S., Kunkel-Bagden, E., Schnell, L., Dai, H.N., Gao, D., Schwab, M.E., 1995. Recovery from spinal cord injury mediated by antibodies to neurite growth inhibitors. Nature 378, 498-501.

Brook, G.A., Houweling, D.A., Gieling, R.G, Hermanns, T, Joosten, E.A., Bar, D.P., Gispen, W.H., Schmitt, A.B., Leprince, P., Noth, J., Nacimiento, W., 2000. Attempted endogenous tissue repair following experimental spinal cord injury in the rat: involvement of cell adhesion molecules L1 and NCAM? Eur. J. Neurosci. 12, 3224-3238.

Bunge, R.P., Puckett, W.R., Hiester, E.D., 1997. Observations on the pathology of several types of human spinal cord injury, with emphasis on the astrocyte response to penetrating injuries. Adv. Neurol. 72, 305-315.

Burin, H.J., Woltmann, G, Grigg, J., 2002. Applicability of laser scanning cytometry to study paediatric alveolar macrophages. Eur. Respir. J. 20, 1437-1443

Chuah, M.I., Choi-Lundberg, D., Weston, S., Vincent, A.J., Chung, R.S., Vickers, J.C., West, A.K., 2004. Olfactory ensheathing cells promote collateral axonal branching in the injured adult rat spinal cord. Exp. Neurol. 185, 15-25.

Coumans, J.V., Lin, T.T., Dai, H.N., MacArthur, L., McAtee, M., Nash, C, Bregman, B.S., 2001. Axonal regeneration and functional recovery after complete spinal cord transection in rats by delayed treatment with transplants and neurotrophins. J. Neurosci. 21, 9334-9344.

Deumens, R., Koopmans, G.C., Den Bakker, C.G., Maquet, V, Blacher, S., Honig, W.M., Jerome, R., Pirard, J.P., Steinbusch, H.W, Joosten, E.A., 2004. Alignment of glial cells stimulates directional neurite growth of CNS neurons in vitro. Neuroscience 125, 591-604.

Deumens, R., Koopmans, G.C., Joosten, E.A., 2005. Regeneration of descending axon tracts after spinal cord injury. Prog. Neurobiol. 77, 57-89.

Dusart, I., Schwab, M.E., 1994. Secondary cell death and the inflammatory reaction after dorsal hemisection of the rat spinal cord. Eur. J. Neurosci. 6, 712-724.

Fawcett, J.W. 1997. Astrocytic and neuronal factors affecting axon regeneration in the damaged central nervous system. Cell Tissue Res. 290, 371-377. 
Franceschini, LA., Barnett, S.C., 1996. Low-affinity NGF-receptor and E-N-CAM expression define two types of olfactory nerve ensheathing cells that share a common lineage. Dev. Biol. 173, 327-343.

Goldshmit, Y., Galea, M.P., Wise, G, Bartlett, P.F., Turnley, A.M., 2004. Axonal regeneration and lack of astrocytic gliosis in EphA4 deficient mice. J. Neurosci. 24, 10064-10073.

GrandPre, T, Li, S., Strittmatter, S.M., 2002. Nogo-66 receptor antagonist peptide promotes axonal regeneration. Nature 417, 547-551.

Grill, R., Blesch, A., Gage, F.H., Tuszynski, M.H., 1997. Cellular delivery of neurotrophin-3 promotes corticospinal axonal growth and partial functional recovery after spinal cord injury. J. Neurosci. 17, 5560-5572.

Hamers, F.P, Lankhorst, A.J., van Laar, T.J., Veldhuis, W.B., Gispen, W.H., 2001. Automated quantitative gait analysis during overground locomotion in the rat: its application to spinal cord contusion and transection injuries. J. Neurotrauma 18, $187-201$.

Imaizumi, T, Lankford, K.L., Kocsis, J.D., 2000. Transplantation of olfactory ensheathing cells or Schwann cells restores rapid and secure conduction across the transected spinal cord. Brain Res. 854, 70-78.

Iwashita, Y., Crang, A.J., Blakemore, W.F., 2000. Redistribution of bisbenzimide Hoechst 33342 from transplanted cells to host cells. NeuroReport 11,1013-1016.

Joosten, E.A., 1997. Corticospinal tract regrowth. Prog. Neurobiol. 53, 1-25. Joosten, E.A., Gribnau, A.A., 1989. Astrocytes and guidance of outgrowing corticospinal tract axons in the rat. An immunocytochemical study using anti-vimentin and anti-glial fibrillary acidic protein. Neuroscience $31,439-452$

Joosten, E.A., Veldhuis, W.B., Hamers, F.P., 2004. Collagen containing neonatal astrocytes stimulates regrowth of injured fibers and promotes modest locomotor recovery after spinal cord injury. J. Neurosci. Res. 77, 127-142.

Koopmans, G.C., Deumens, R., Honig, W.M., Hamers, F.P., Steinbusch, H.W., Joosten, E.A., 2005. The assessment of locomotor function in spinal cord injured rats: the importance of objective analysis of coordination. J. Neurotrauma 22, 214-225.

Li, Y, Field, P.M., Raisman, G, 1997. Repair of adult rat corticospinal tract by transplants of olfactory ensheathing cells. Science 277, 20002002 .

Li, Y, Field, P.M., Raisman, G, 1998. Regeneration of adult rat corticospinal axons induced by transplanted olfactory ensheathing cells. J. Neurosci. 18, 10514-10524

Li, Y, Decherchi, P., Raisman, G, 2003. Transplantation of olfactory ensheathing cells into spinal cord lesions restores breathing and climbing. J. Neurosci. 23, 727-731.

Li, Y, Field, P.M., Raisman, G, 2005. Olfactory ensheathing cells and olfactory nerve fibroblasts maintain continuous open channels for regrowth of olfactory nerve fibres.

Glia 52, 245-251. Lu, J., Feron, F., Ho, S.M., Mackay-Sim, A., Waite, P.M., 2001. Transplantation of nasal olfactory tissue promotes partial recovery in paraplegic adult rats. Brain Res. 889, 344-357.

Mainil-Varlet, P., Curtis, R., Gogolewski, S., 1997. Effect of in vivo and in vitro degradation on molecular and mechanical properties of various low-molecular-weight polylactides. J. Biomed. Mater. Res. 36, 360-380. Maquet, V, Martin, D., Scholtes, F., Franzen, R., Schoenen, J., Moonen, G, Jerme, R., 2001. Poly(D,L-lactide) foams modified by poly(ethylene oxide)-block-poly(D,L-lactide) copolymers and aFGF: in vitro and in vivo evaluation for spinal cord regeneration. Biomaterials 22, 1137-1146.

Nash, H.H., Borke, R.C., Anders, J.J., 2002. Ensheathing cells and methylprednisolone promote axonal regeneration and functional recovery in the lesioned adult rat spinal cord. J. Neurosci. 22, 7111-7120.

Oudega, M., Vargas, C.G, Weber, A.B., Kleitman, N, Bunge, M.B., 1999. Long-term effects of methylprednisolone following transection of adult rat spinal cord. Eur. J. Neurosci. 11, 2453-2464.

Pallini, R., Fernandez, E., Sbriccoli, A., 1988. Retrograde degeneration of corticospinal axons following transection of the spinal cord in rats. A quantitative study with anterogradely transported horseradish peroxidase. J. Neurosurg. 68, 124-128.

Pasterkamp, R.J., Giger, R.J., Ruitenberg, M.J., Holtmaat, A.J., De Wit, J., De Winter, F., Verhaagen, J., 1999. Expression of the gene encoding the chemorepellent semaphorin III is induced in the fibroblast component of neural scar tissue formed following injuries of adult but not neonatal CNS. Mol. Cell. Neurosci. 13, 143-166.

Perez-Bouza, A., Wigley, C.B., Nacimiento, W, Noth, J., Brook, G.A., 1998. Spontaneous orientation of transplanted olfactory glia influences axonal regeneration. NeuroReport 9, 2971-2975.

Plant, G.W. Christensen, C.L., Oudega, M., Bunge, M.B., 2003. Delayed transplantation of olfactory ensheathing glia promotes sparing/regeneration of supraspinal axons in the contused adult rat spinal cord. J. Neurotrauma 20, 1-16. 
Published in: Experimental Neurology (2006), vol. 200, iss. 1, pp. 89-103

Status: Postprint (Author's version)

Raisman, G, 2001. Olfactory ensheathing cells-Another miracle cure for spinal cord injury? Nat. Rev., Neurosci. 2, $369-375$.

Ramon-Cueto, A., Avila, J., 1998. Olfactory ensheathing glia: properties and function. Brain Res. Bull. 46, $175-187$.

Ramon-Cueto, A., Nieto-Sampedro, M., 1992. Glial cells from adult rat olfactory bulb: immunocytochemical properties of pure cultures of ensheathing cells. Neuroscience 47, 213-220.

Ramon-Cueto, A.,Cordero, M.I., Santos-Benito, F.F., Avila, J., 2000. Functional recovery of paraplegic rats and motor axon regeneration in their spinal cords by olfactory ensheathing glia. Neuron $25,425-435$.

Richardson, P.M., Issa, V.M., Aguayo, A.J., 1984. Regeneration of long spinal axons in the rat. J. Neurocytol. 13, $165-182$.

Sasaki, M., Lankford, K.L., Zemedkun, M., Kocsis, J.D., 2004. Identified olfactory ensheathing cells transplanted into the transected dorsal funiculus bridge the lesion and form myelin. J. Neurosci. 24, 8485-8493.

Schnell, L., Schwab, M.E., 1990. Axonal regeneration in the rat spinal cord produced by an antibody against myelin-associated neurite growth inhibitors. Nature 343, 269-272.

Schnell, L., Schwab, M.E., 1993. Sprouting and regeneration of lesioned corticospinal tract fibres in the adult rat spinal cord. Eur. J. Neurosci. 5, 1156-1171.

Schnell, L., Schneider, R., Kolbeck, R., Barde, Y.A., Schwab, M.E., 1994. Neurotrophin-3 enhances sprouting of corticospinal tract during development and after adult spinal cord lesion. Nature 367, 170-173.

Starkey, M.L., Hamers, F.F., Doherty, P., McMahon, S.B., Bradbury, E.J., 2004. Assessing behavioural function following unilateral lesion of the corticospinal tract in adult mice. Program No 43102004 Abstract Viewer/Itinerary Planner. Society for Neuroscience, Washington, DC.

von Meyenburg, J., Brosamle, C, Metz, G.A., Schwab, M.E., 1998. Regeneration and sprouting of chronically injured corticospinal tract fibers in adult rats promoted by NT-3 and the mAb IN-1, which neutralizes myelin-associated neurite growth inhibitors. Exp. Neurol. 154, 583-594.

Wagner Jr., F.C., VanGilder, J.C., Dohrmann, G.J., 1978. Pathological changes from acute to chronic in experimental spinal cord trauma. J. Neurosurg. 48, 92-98

Woodhouse, A., Vincent, A.J., Kozel, M.A., Chung, R.S., Waite, P.M., Vickers, J.C., West, A.K., Chuah, M.I., 2005. Spinal cord tissue affects ensheathing cell proliferation and apoptosis. NeuroReport 16, 737-740. 